\title{
How Serious is ADHD?
}

\section{Robert Eme ${ }^{1}$}

${ }^{1}$ Illinois School of Professional Psychology at Argosy University, Schaumburg Campus, USA

${ }^{*}$ Corresponding author: Robert Eme, Illinois School of Professional Psychology at Argosy University, Schaumburg Campus, USA; Tel: 8479694949; E-mailreme@argosy.edu

Received date: August 11, 2017, Accepted date: August 13, 2017, Published date: August 20, 2017

Copyright: (c) 2017 Eme R. This is an open-access article distributed under the terms of the Creative Commons Attribution License, which permits unrestricted use, distribution, and reproduction in any medium, provided the original author and source are credited.

\begin{abstract}
Even though there are well over 20,000 studies of Attention-Deficit/Hyperactivity Disorder (ADHD), it is still viewed with skepticism by some and often remains stigmatized by the media. Hence, given the continuing challenges to the validity of ADHD, this article provided a concise overview of the evidence that establishes ADHD as brain disorder associated with a set of well-defined symptoms that causes serious impairment in many life activities.
\end{abstract}

Keywords Attention-deficit/hyperactivity disorder; Brain disorder; Physical health

\section{Introduction}

Even though there are well over 20,000 studies of Attention-Deficit/ Hyperactivity Disorder (ADHD) dating from 1775, with at least 800-1000 new journal articles appearing annually, there continue to be media flare-ups portraying $\mathrm{ADHD}$ as a minor disorder or no disorder at all $[1,2]$. As Thapar and Cooper [3] remarked: "...ADHD is still viewed with skepticism by some and often remains stigmatized by the media..." For example, most recently the book ADHD Nation depicts ADHD as largely a creation of the pharmaceutical industry fueling an epidemic of over-diagnoses. In short, it is alleged that ADHD is largely, if not completely, the medicalizing of the typical characteristics of being a child, especially a boy [4]. Hence, given the continuing challenges to the validity of ADHD, there is a need for an article that will provide a concise overview of the evidence that establishes ADHD as a valid mental disorder defined as a condition associated with a set of well-defined symptoms that causes serious impairment [1]. Since the literature on ADHD is enormous, the article will of necessity draw on the conclusions of several current, comprehensive reviews, with examples from recent studies $[1,3,4,5,6,7,8,9]$. Following a brief introductory overview of ADHD, the paper will discuss the evidence that ADHD is a brain disorder which provides the best evidence of its validity as a real disorder. Indeed, this evidence has been found to be so decisive that ADHD has been included in the category of neurodevelopment disorders by the American Psychiatric Association [10] which by definition designates a disability involving some form of disruption to brain development [11]. The paper will then establish that the real brain disorder of ADHD increases risk for serious impairment in the three major domains of mental health, educational performance, and social functioning. There are impairments in other domains such as physical health and occupation [1], but a discussion of the three domains is sufficient to establish the gravity of ADHD.

\section{What is ADHD?}

ADHD, with a world-wide prevalence in the general juvenile population of $7 \%$ (male/female ratio of $3: 1$ ) [12,13], is the diagnosis of a neurodevelopmental disorder with typical onset in childhood given to individuals who present with significant, impairing symptoms of inattention, impulsivity, and hyperactivity [10]. These impairments persist into adolescence and adulthood for a majority of individuals $[2,3,6,8]$. The three main "presentations" of ADHD in the Diagnostic and Statistical Manual of Mental Disorders-5 (DSM-5) are: predominantly inattentive, predominantly hyperactive/impulsive, and the combined form when criteria are met for both inattentive and hyperactive/impulsive symptoms [10]. Although ADHD, along with all other conditions in the DSM, is considered as a diagnostic category, there is a strong consensus that ADHD, as well as many other childhood disorders is best conceptualized in dimensional rather than categorical terms $[3,5,7,9]$. Namely, the presentations of ADHD are best understood as representing two distinct, albeit highly-related spectra or dimensions of inattention/disorganization and hyperactivity/impulsivity on which individuals differ [3,5,7,9]. Thus, ADHD can be conceptualized as both a diagnostic category which is useful given that clinical decisions tend to be categorical in nature (e.g., the individual either does or does not have a disorder that requires treatment), and a set of behavioral dimensions which more accurately reflects the reality of most childhood mental disorders and many medical disorders such as diabetes or hypertension [3].

ADHD symptoms are caused by dysfunction in multiple psychological processes which can be linked to multiple abnormalities in the brain's neural circuits that undergird these processes.

\section{Abnormal Brain Functioning}

The strongest evidence that ADHD is a real disorder and not simply a medicalizing of normal juvenile behavior comes from the numerous studies that have established that ADHD is biologically based brain disorder. These studies can be grouped into two major categories: genetics and neuroimaging (structural and functional) studies of the brain. Curiously, as noted by Zametkin and Solanto [14] in their review of ADHD Nation, Schwartz omits virtually all reference to this research.

Regarding genetics, as with most complex disorders, although no single risk factor is either sufficient or necessary to explain ADHD, ADHD is a highly genetically influenced disorder such that $70 \%$ to $80 \%$ of the variation in symptoms is explained by genetic factors $(2,3,7,9]$. Despite this substantial heritability, the identification of 
Page 2 of 4

ADHD risk genes has yielded "disappointingly small results to date" [9]. This is most likely due to ADHD having a complex, polygenetic background, in which multiple gene variants (i.e., hundreds, many with small effects) contribute to the etiology of disorder, therefore making it very difficult to detect an effect of a single gene.

These genetic factors have been linked multiple structural (e.g., smaller volume) and functional (e.g., underactivation, impaired connectivity) brain abnormalities in individuals with ADHD which compromise the functioning of neural circuits related to attention, inhibitory control, reward motivation, and emotional regulation and which in turn can be linked to ADHD symptoms and consequent impairments in major life activities $[3,7,9,15,16,17]$. However, although the evidence for these linkages is robust, the research is correlational and therefore cannot be interpreted as causal on the sole basis of the linkage [16]. Nevertheless, the fact that stimulant medication has been found to increase activity in these same regions $[18,19]$ consistent with therapeutic effects on symptoms and impairments that have been described as "night and day," [20] offers significant support for a causal interpretation.

The evidence documenting the impairments in three major domains will now be presented, followed by a discussion of a dysfunctional neural circuit and the psychological process they subserve that plays a major role in the etiology of the impairment. Note that the linkage between the impairment and the neural circuit is meant to highlight the reality of ADHD as a brain disorder, and is not meant to imply that the dysfunctional neural circuit is the only factor (biological or psychosocial) contributing to the impairment.

\section{Educational Impairments}

Juveniles with ADHD are at greater risk than their non-ADHD peers for poor academic achievement, grade repetition, dropping out, and fewer years of education [1]. There is a worsening of achievement as the academic challenges become greater with the transition from elementary to middle school or high school. More recent studies however have found a decrease in the dropout rate with $10 \%$ or fewer of juveniles with ADHD failing to complete high school compared with earlier rates of $25 \%$ to $40 \%$ [2]. This decline is most likely due to the greater access to special education services than in the past.

\section{Dysfunctional Neural Circuit}

Educational impairments have been found to be more a function of symptoms in inattention/disorganization dimension than the hyperactive/impulsive dimension [1]. Attention can be defined as the processing of one piece or source of information over others - in other words, the ability to focus or filter information [9]. Obviously, a defective filter can make it very difficult to process the information needed for academic success. In the words of a young adult with ADHD: "The problem that dominated my life and shaped my personality was the need to avoid the piercing, rasping, blasting, disorganized chaos of incoming stimuli that I could not filter out, could not ignore. This made it very hard for me to relate to other people: to think, study, make it in school, to carry out tasks, plan ahead, and remember" [21]. Specific aspects of attention (orienting, arousal, and executive control) have been linked to specific networks $[9,16]$. The aspect of attention related to a defective filter is executive attention which involves the voluntary control of behavior in accordance with goals [16] and has been linked to a dysfunctional frontoparietal circuit which includes the dorsoslateral prefrontal cortex, the interparietal sulcus, and the medial cingulate cortex.

\section{Mental Health Impairments}

Several longitudinal studies that have followed up children with ADHD into adolescence and adulthood have concluded that the disorder puts the children at increased risk for several mental disorders. Among the various disorders, the strongest link is with sequential development of disorders in the externalizing spectrum, i.e., Oppositional Defiant Disorder, Conduct Disorder, Substance Use Disorder, and Antisocial Personality Disorder [8,15,22]. This developmental cascade in turn increases the risk for juvenile delinquency and adult criminality such that a systematic review and meta-analysis of 11 prospective studies $(\mathrm{N}=15,442)$ found that childhood ADHD was significantly associated with increased risk for juvenile arrests (RR: 2.2, 95\%, CI: 1.3-3.5),convictions (RR: 3.3, 95\%, CI: 2.1-5.2), and incarcerations (RR: 2.9, 95\%, CI: 1.9-4.3) [23]. Unsurprisingly therefore, a meta-analysis of 42 international studies of the prevalence of $\mathrm{ADHD}$ in incarcerated populations reported a prevalence of $21.7 \%$ which is three times greater than the prevalence rate in the general population [24]. A recent example of the risk ADHD poses for one of the disorders in the externalizing spectrum comes from a study by Harvey, Breaux, and Lugo-Candelas [25] which sought to advance the understanding of how to explain the substantial comorbidity between ADHD and Oppositional Defiant Disorder (ODD) that develops during the preschool years such that between one third and one half of children who are diagnosed with one disorder are also comorbid for the other disorder. Participants were 199 children (107 boys) who were recruited from the community for a longitudinal study of preschoolers with behavior problems. Parental reports of ADHD and ODD symptoms were collected annually from ages 3 to 6 and a family history interview was administered at age 3 . The results provided strong support for a developmental precursors model to explain the comorbidity. Namely, ADHD was a strong predictor for the development of the argumentative/defiant symptoms of ODD. This progression from ADHD to ODD is best explained by the ADHD symptoms of behavioral and emotional impulsivity which greatly increase the risk for coercive, oppositional interchanges with significant others in the child's life $[15,26,27]$. Indeed, it is estimated that a typical child with ADHD has an astonishing half a million of these negative interchanges each year [28].

\section{Dysfunctional Neural Circuit}

The increased risk for the development of disorders in the externalizing spectrum is conferred by the behavioral impulsivity of the hyperactive/impulsive and combined presentations of ADHD. Behavioral impulsivity is characterized by: a) decisions based on a preference for smaller, immediate rewards over larger, delayed rewards, and b) a tendency to make rapid decisions without forethought, adequate consideration of the evidence [15]. Current comprehensive reviews concur in implicating impairments in the mesolimbic and mesocortical dopamine (DA) neural circuits and their interconnections to one another as well as other brain regions as the central neurobiological mechanisms in generating behavioral impulsivity $[9,15,29]$. These two circuits are linked to two different aspects of impulsivity: impulse generation and impulse inhibition.

Mesolimbic DA circuit and impulse generation: The subcortical mesolimbic DA projections ascend from the ventral tegmental area to the ventral striatum, including the nucleus accumbens and the ventral 
regions of the caudate nucleus and putamen [30]. Several lines of research indicate that this circuit is the brain's most important reward system [15,30]. Subsequently, extensive neuroimaging research has found reduced mesolimbic activity during the anticipation of reward in individuals with ADHD [15,30]. This hypofunctioning of the mesolimbic circuit results in an undersensitivity to reinforcing stimuli which is hypothesized to trigger impulsivity in two ways. First, undersensitivity leads to temporal discounting of reward in individuals with ADHD who find the experience in delay of reinforcement so aversive that they tend to impulsively choose immediate rewards even when an alternative option would result in larger reward after a longer delay. Second, under sensitivity leads to excessive, impulsive reward seeking as individuals with ADHD "experience relatively low hedonic value from pleasurable stimuli and therefore seek more frequent extreme incentives to derive hedonic payoff" [30]. In short, under sensitivity to stimuli that most individuals would find reinforcing results in individuals with ADHD having chronic feelings of anhedonia which they find aversive. To alleviate such feelings, they tend to impulsively seek immediate and frequent reinforcement/gratification.

Mesocortical DA circuit and impulse inhibition: The mesocortical DA circuit involves neural projections from the ventral tegmental area to cerebral cortex, and the prefrontal cortex (PFC)and the anterior cingulate cortex $[9,15,29]$. This circuit is involved with the second aspect of impulsivity, i.e., how impulses are inhibited [9]. The main substructures of the PFC are the dorsolateral PFC; ventromedial PFC; ventrolateral PFC; the orbitofrontal PFC; and anterior cingulate cortex. Each of these substructures are hypothesized to be somewhat differentially involved in top down impulse control [16,29]. Several lines of research document that a breakdown in optimal function and communication between the PFC and the midbrain region can result in failures to contain, inhibit, interrupt impulsive behavior $[9,15,16,29]$.

\section{Social Functioning}

Comprehensive literature reviews have firmly established that ADHD is associated with impaired relationships with parents and peers. Parent-child relations are characterized by high levels of conflict, oppositionality, and parenting stress [31]. Peer relations, especially in the combined and predominantly hyperactive/impulsive presentations, display high rates of intrusive, annoying and disruptive behavior resulting in children with ADHD being more likely to be rejected and bullied by their peers [32]. A major contributor to conflict with parents and disruptive behavior with peers is emotional dysregulation. For most of the 170 years of its medical history ADHD was believed to involve deficits in emotional regulation. Namely, individuals with ADHD are highly likely to manifest low frustration tolerance, impatience, quickness to anger, and are easily aroused to emotional reactions more generally. Then "inexplicably" [2], with the appearance of DSM-II in the early 1960's, "Emotional dysregulation has been ignored in descriptions of and diagnostic criteria for the disorder" and considered as merely an associated problem that may arise in some cases. However, there is now compelling evidence that strongly argues for emotional impulsivity to be returned as a core component of ADHD [26]. For example, most recently a study found that deficits in emotional regulation documented in older children with ADHD are already evident in younger children (ages 4 to 7 ), and distinct from the developmentally appropriate emotional dysregulation typically seen this young age group [33]. In short, although there is still not complete agreement among researchers, it appears increasingly likely emotional impulsivity is as an inherent a part of the broader hyperactive/ impulsive dimension of ADHD as is behavioral impulsivity.

\section{Dysfunctional Neural Circuit}

Although the amygdala has long been referred to as the seat of emotion, it is now well known that there is a neural network working in concert with the amygdala to process emotion [34]. The primary nodes in this network involve the orbitofrontal cortex and its connections with the amygdala and ventral striatum [35,36]. As reviewed by Shaw and colleagues [35], prior research has found structural abnormalities in the amygdala and weaker connectivity between the amygdala and the orbitofrontal cortex in individuals with ADHD. Most recently, and most impressively, the finding of structural abnormalities in the amygdala has received resounding confirmation in a mega-analysis in the largest data set to date of subcortical brain volume differences in individuals with $\mathrm{ADHD}$ and comparison subjects, i.e., 1713 individuals with ADHD and 1529 controls [17,37]. For the first time ever, the study found reduced volume in the ventral striatum and the amygdala (which showed the largest effect size of all the subcortical structures tested) in children with ADHD, thereby providing strong support for emotional dysregulation as a core symptom of ADHD. Lastly, the authors' comments on the overarching significance of their findings warrants quoting in full, as it perfectly captures and validates the goal of this article.

...we have no doubt that we all share the desire not to return to the relatively recent days (italics added) when individuals with ADHD were dismissed as lazy, having bad parents, or being bad children. Understanding of ADHD as a brain disorder (italics added) and clarifying the extent of its biological origins has helped direct focus away from moral judgments towards developing evidence based treatment of the impairing symptoms of the disorder [38].

\section{Conclusion}

The evidence adduced in this article to support the validity of ADHD as a real brain disorder which increases the risk of serious impairment in many of life's activities along with the 20,000 plus studies of ADHD since its first recognition as an impairing condition serve as a massive refutation of the still re-occurring canard that ADHD is largely a bogus disorder. It is a real disorder which requires treatment and should not be derisively dismissed as the medicalizing of lazy, bad children and bad parents.

\section{References}

1. Barkley R (2015a) Attention-deficit hyperactivity disorder: A handbook for diagnosis \& treatment 4 .

2. Barkley R (2016) Recent longitudinal studies of childhood attentiondeficit hyperactivity disorder: Important themes and questions for further research. J Abnorm Psychol 125: 248-255.

3. Thapar A and Cooper M (2016) Attention deficit hyperactivity disorder. The Lancet 387: 1240-1250.

4. Hinshaw $S$ and Ellison K (2016) ADHD: What everyone needs to know.

5. Ahmad S and Hinshaw S (2016) Attention-deficit/hyperactivity disorder: Similarities to and differences from other externalizing disorders. The Oxford handbook of externalizing spectrum disorders 19-37.

6. Asherson P, Buitelaar J, Faraone S \& Rohde L (2016) Adult attentiondeficit hyperactivity disorder: key conceptual issues. Lancet Psychiatry 3: 568-578. 
7. Faraone S, Asherson P, Banashewski, Biederman J, Buitelarr J, et al. (2015) Attention-Deficit/hyperactivity Disorder. Nature review disease primers 1: 1-23.

8. Hechtman L (2017) Attention Deficit Hyperactivity Disorder: Adult outcome and its predictors.

9. Nigg J (2017) Attention-Deficit/Hyperactivity Disorder. J Clin Child Adolesc Psychol 3: 407-448.

10. American Psychiatric Association (2013) Diagnostic and statistical manual of mental Disorders 5 .

11. Thapar A, Cooper M and Rutter M (2017) Neurodevelopmental disorders. Lancet Psychiatry 4: 339-346.

12. Owens E, Cardoos S and Hinshaw S (2015) Developmental progression and gender differences in individuals with ADHD. Attention-deficit/ hyperactivity disorder: A handbook for diagnosis and treatment 4 : 223-255.

13. Thomas R, Sanders S, Doust J, Beller E and Glasziou P (2015) Prevalence of attention deficit hyperactivity disorder: A systematic review of metaanalysis. Pediatrics 135: e994-e1001.

14. Zametkin A and Solanto M (2017) A review of ADHD Nation. The ADHD Report 1: 6-10.

15. Beauchaine T, Zisner A and Sauder C (2017) Trait impulsivity and the externalizing spectrum. Annu rev psychol 13: 343-368.

16. Gallo E and Posner J (2016) Moving towards causality in attention-deficit hyperactivity disorder. Lancet Psychiatry 3: 555-567.

17. Hoogman M, Bralten J, Hibar D, Messes M, Zwiers M, et al. (2017a) Subcortical brain volume differences in participants with attention deficit hyperactivity disorder in children and adults: a cross-sectional megaanalysis. The Lancet 4: 310-319.

18. Rubia K., Alegria A and Brinson H (2014) Brain abnormalities in attention-deficit hyperactivity disorder: A review. Rev Neurol 58: S3-16.

19. Spencer T, Brown A, Seidman L, Valera E, Makris N, et al. (2013) Effects of psychostimulants on brain structures and function in ADHD: A qualitative literature of magnetic resonance imaging-based neuroimaging studies J Clin Psychiatry 74: 902-917.

20. Hinshaw S and Scheffler R (2014) The ADHD explosion.

21. Miller D and Blum K. (1996) Overload-Attention deficit disorder and the addictive brain.

22. Beauchaine $\mathrm{T}$ and Hinshaw S (2016) The Oxford handbook of externalizing spectrum disorders.

23. Jensen MC and Steinhausen H (2016) A meta-analysis and systematic review of the risks associated with childhood-attention deficit/ hyperactivity disorder on long-term outcome of arrests, convictions, and incarcerations. Clin Psychol Rev 48: 32-42.
24. Young S, Moss D, Sedgwick O, Fridman M and Hodgkins P (2015) A meta-analysis of the prevalence of attention-deficit/hyperactivity disorder in incarcerated populations. Psychol Med 45: 247-258.

25. Harvey E, Breaux R and Candelas LC (2016) Early development of comorbidity between symptoms of Attention-Deficit/Hyperactivity Disorder (ADHD) and Oppositional Defiant Disorder (ODD). J Abnorm Psychol 125: 154-167.

26. Barkley R (2015b) Emotional dysregulation is a core component of ADHD. Attention-deficit hyperactivity disorder: A handbook for diagnosis \& treatment 4: 81-115.

27. Snyder J (2016) Coercive family processes in the development of externalizing behavior: incorporating neurobiology into intervention research. The Oxford handbook of spectrum disorders 286-302.

28. Pelham W and Fabiano G (2008) Evidence based psychosocial treatments for attention deficit/hyperactivity disorder. J Clin Child Adolesc Psychol 37: 184-214.

29. Ryan CN and Seguin J (2016) Prefrontal and anterior cingulate cortex mechanisms of impulsivity. Oxford handbook of externalizing spectrum disorders 184-200.

30. Zisner A and Beauchaine $\mathrm{T}$ (2016) Midbrain mechanisms of trait impulsivity. Oxford handbook of externalizing spectrum disorders 184-200.

31. Johnston C and Tuscano CA (2015) Families and ADHD. Attentiondeficit hyperactivity disorder: A handbook for diagnosis \& treatment 4 : 191-209.

32. McQuade J and Hoza B (2015) Peer relationships of children with ADHD. Attention-deficit hyperactivity disorder: A handbook for diagnosis \& treatment 4: 210-222.

33. Candelas LC, Flegenheimer C, McDermott J and Harvey E (2016) Emotional understanding, reactivity, and regulation in young children with ADHD symptoms. J Abnorm Child Psychol.

34. Stujenske J and Likhtik E (2017) Fear from the bottom up. Nature Neuroscience 20: 765-767.

35. Shaw P, Stringaris A, Nigg J and Leibenluft E (2014) Emotional dysregulation and attention-deficit/hyperactivity disorder. Am J Psychiatry 171: 276-293.

36. Barkley R (2015c) Health problems and related impairments in children and adults with ADHD. Attention-deficit hyperactivity disorder: A handbook for diagnosis \& treatment 4: 267-313.

37. Nigg J (2016) Attention and impulsivity. Developmental psychopathology: Risk, disorder and adaptation 3: 591-646.

38. Hoogman M, Bralten J, Hibar D, Messes M, Zwiers M, et al. (2017b) Authors reply. The Lancet, 4: 440-441. 